\title{
Le Bras, H. (2016), Anatomie sociale de la France, París, Robert Laffont
}

\author{
Eguzki Urteaga*
}

Hervé Le Bras acaba de publicar su más reciente obra, titulada Anatomie sociale de la France (Anatomía social de Francia), en la editorial Robert Laffont, cuya colección Le monde comme il va está dirigida por Michel Wieviorka. Es preciso recordar que este historiador y demógrafo galo, titular de la cátedra Territorios y Poblaciones del Colegio de Estudios Mundiales, de la Fundación Casa de las Ciencias Humanas (FMSH), es director de investigación emérito en el Instituto Nacional de Estudios Demográficos (INED) y director de estudios en la prestigiosa Escuela de Altos Estudios en Ciencias Sociales (EHESS). Entre sus obras más recientes podemos citar: Le mystère français (2013), escrita con Emmanuel Todd; L'atlas des inégalités (2014); Le Pari du FN (2015), y Le nouvel ordre électoral (2016). Esa labor investigadora ha sido recompensada con varios galardones y premios tales como la Legión de Honor.

En la presente obra, Le Bras observa que las encuestas por sondeo ofrecen una imagen fragmentada de la ciudadanía gala dado que analizan sus rasgos particulares según un orden preciso. El problema es que las personas se distinguen por tener todas estas peculiaridades a la vez, de modo que no puedan ser reducidas a estos atributos (p. 9). Además, "sus comportamientos dependen tanto de sus entornos próximos como de sus propias características. Por lo tanto, para comprenderlas es necesario disponer de una masa enorme de informaciones que permitan cruzar las numerosas características individuales y las de sus familiares" (p. 9).

Para el autor, el censo responde a esta exigencia, de forma que los datos provenientes del que se realizó en 2011 constituyan la base empírica de esta obra. "Gracias a los datos individuales y, sobre todo, gracias a las características de los hogares y de sus localizaciones $[\ldots]$ permite sondear detalladamente las conductas de los franceses" (pp. 9-10). De hecho, a lo largo del libro se privilegian tres dimensiones sociodemográficas: la composición de las parejas, la inmigración y el desempleo. Estas variables son cruzadas entre sí con otros criterios y, especialmente, con el nivel educativo, la clase

* Universidad del País Vasco, Departamento de Sociología y Trabajo Social, Facultad de Relaciones Laborales y Trabajo Social. Dirección postal: Los Apraiz, 2, ES-01006, Vitoria. Correo electrónico: <eguzki.urteaga@ehu.eus>.

ESTUDIOS DEMOGRÁFICOS Y URBANOS, VOL. 32, NÚM. 2 (95), 2017, pp. 425-433 
social, el tamaño de la familia y el lugar de residencia (p. 10). La imagen que resulta de todo ello es más sofisticada y compleja que la que ofrecen los sondeos de opinión.

En cuanto a la estructura de esta obra, "los capítulos de este libro [proceden] por círculos concéntricos en torno a los individuos. [Parten] de los atributos individuales de edad, categoría social, nivel educativo y origen nacional. Posteriormente, se [interesan] por las características del eventual cónyuge, de la familia, del hogar e incluso del parentesco. Finalmente, en el círculo más amplio, las peculiaridades de los lugares de residencia de los individuos son integradas en el análisis" (p. 10).

En el capítulo inicial, dedicado a la anatomía social de las parejas galas, el autor se interesa por las profesiones ejercidas por los hombres y las mujeres. Observa que si ellas han incrementado notablemente sus tasas de actividad, lo han hecho en ámbitos separados, dado que el reparto de las preferencias sociales es muy estable (p. 20). En efecto, existe "una estructura social profunda y estable de la sociedad que ha resistido a los numerosos cambios educativos, industriales, políticos y morales que Francia ha conocido a lo largo de los últimos cuarenta años" (p. 21).

Esa constancia se vislumbra igualmente en cuanto a la clase social, ya que hay una mayor probabilidad de que una persona tenga un cónyuge perteneciente a la misma categoría social, lo que no resulta tanto de una intención deliberada, sino de las posibilidades objetivas de encontrarse en entornos sociales delimitados. A menudo, "los futuros cónyuges han realizado las mismas carreras universitarias, han participado en las mismas fiestas, los mismos lugares de trabajo o de ocio, y han practicado los mismos deportes" (p. 24). Sin embargo se observan algunos matices. Así, los cuadros directivos tienen cierta tendencia a emparejarse en mayor medida que el resto de la población francesa con personas pertenecientes a su categoría social y "su probabilidad de emparejarse con un obrero o una obrera es escasa" (p. 28).

En cuanto al nivel educativo, los datos del censo indican que, mientras que en las parejas más envejecidas tanto hombres como mujeres contraen matrimonio con personas del mismo nivel educativo o de uno inmediatamente inferior o superior, no sucede exactamente lo mismo con las nuevas generaciones, dado que esta correlación no es tan fuerte (p. 32). Efectivamente, entre las nuevas generaciones todos los niveles inferiores al bachillerato forman una masa indecisa en el seno de la cual no se elige cónyuge en función de su nivel educativo. Pero a partir del bachillerato todo cambia. Además, la elección de los cónyuges varía sobremanera según el género, dado que los hombres eligen raramente como pareja a una mujer menos calificada, mientras que las mujeres son más tolerantes y abiertas al respec- 
to (p. 32). Para Le Bras, la principal razón de esa diferencia y de ese cambio estriba en la inversión rápida de los niveles educativos entre hombres y mujeres, lo que tiene como consecuencia que, hoy en día, los hombres tengan en promedio una cónyuge más diplomada que ellos y suceda todo lo contrario con las mujeres (p. 34).

Según la lógica meritocrática, el reparto de hombres y mujeres por categoría social debería haberse modificado con el transcurso del tiempo. Pero no es lo que sucede, puesto que la estructura social no ha seguido la evolución de la estructura educativa (p.35). Dado que los títulos académicos inferiores al bachillerato están desvalorizados, han dejado de ser tomados en consideración a la hora de formar pareja. "La razón principal por la cual los hombres conservan su ventaja social es la separación de los roles y, por lo tanto, de las tareas desempeñadas en el seno del hogar" (p. 36). En ese contexto, dado que la obtención de un título académico similar al de los hombres no garantiza a las mujeres la misma posición social que los varones, deben conseguir una calificación superior para compensar su desventaja de género (p. 35).

Para el autor, "el contraste entre la permanencia de las estructuras sociales y la evolución rápida de las estructuras educativas es potencialmente explosiva, [ya que] [...] está, sin lugar a dudas, en el origen de las tensiones políticas que conoce Francia con el auge del Frente Nacional: la notable elevación del nivel educativo entre 1975 y 1995 no ha tenido una traducción en el mercado laboral, provocando desclasificaciones, desempleo y ausencia de perspectivas de carrera profesional, fuentes de un profundo malestar" (p. 36). Parece lógico dado que la elevación del nivel de estudios va de la mano de un incremento de las expectativas sociales. Se observa un fenómeno similar en el seno de las parejas, puesto que, a partir del momento en que la mujer está más educada que su cónyuge, aspira a una elevación social que se plasme en una mejor situación profesional. Puede acceder a ella, bien consiguiendo un reparto más equitativo de las tareas domésticas, o bien reduciendo el número de hijos a su cargo, lo que disminuye automáticamente su implicación doméstica (pp. 36-37). Sucede lo mismo con la inmigración, dado que, si "los inmigrantes de los años 1960 [...] tenían un escaso nivel educativo y una familia que permanecía en el país [de origen] [...], los inmigrantes más recientes tienen de media un buen nivel educativo y han contraído a menudo matrimonios mixtos" (p. 37), lo que se traduce en sus actitudes y expectativas.

En el capítulo siguiente del libro, centrado en las parejas mixtas, el demógrafo galo observa que las uniones mixtas, es decir las parejas formadas por un cónyuge extranjero y otro nacido en Francia o es francés de nacimiento, constituyen el mejor indicador de integración de la población inmigrante 
residente en el Hexágono. Hoy en día, el 35.8\% de los inmigrantes que viven en pareja es de ese tipo, sabiendo que "la proporción de uniones mixtas decrece con la edad, pasando del $64.3 \%$ en la generación más joven ( 20 a 24 años) al $42 \%$ en la de edad más avanzada (60 a 64 años)" (p. 40). Esta progresión de las uniones mixtas se constata igualmente en valores absolutos, puesto que han pasado de 2.8 millones en 1962 a 5.6 millones en 2011 (p. 41). El aumento de la "mixidad" o mezcla étnica a lo largo de los últimos cuarenta años resulta, en gran medida, de la elevación del nivel educativo, en la medida en que esa elevación propicia la multiplicación de las parejas con orígenes y nacionalidades diferentes (p. 44). No en vano ese vínculo varía en función del nivel educativo de los inmigrantes, dado que es efectivo entre los más calificados mientras que no es notablemente menor entre los extranjeros de titulación media y baja.

A su vez, "la 'mixidad' de las parejas depende [...] de las proporciones relativas de inmigrantes y no inmigrantes en cada lugar" (p. 83). De hecho, mientras que en ciertos departamentos galos los inmigrantes adultos que se hallan emparejados representan menos del 3\% del total, en otros esta proporción supera el $25 \%$. En general, Le Bras constata que los inmigrantes están concentrados en las grandes aglomeraciones, a lo largo de las principales vías de circulación y cerca de las fronteras terrestres, "lo que resume parte de la historia y de los factores de inmigración [en Francia]" (p. 83). Así, constata que, en la mayoría de los departamentos en los cuales los inmigrantes son numerosos, la proporción de matrimonios entre inmigrantes es preponderante. En ese sentido, la mayoría de los departamentos de la región parisina, de la región de Lyon, de la zona mediterránea y de Alsacia figuran entre los más endogámicos, con más de la mitad de uniones entre inmigrantes. Al contrario, los departamentos de la fachada atlántica y del norte de Francia son más abiertos a las parejas mixtas (p. 85).

En el capítulo posterior, que efectúa un análisis pormenorizado de la fecundidad, el autor constata que, hoy en día, "agricultores y obreros constituyen las categorías más fecundas, aunque sean alcanzados por los empleados, artesanos y comerciantes. Las clases medias y superiores son las menos fecundas" (p. 108) y, especialmente, los cuadros y las profesiones liberales. Se produce un doble proceso en dirección contraria que afecta a las categorías sociales: uno hacia la convergencia, porque la diferencia entre las categorías extremas se ha reducido notablemente; y otro hacia la divergencia, puesto que se produce una oposición brutal entre las clases medias y superiores, por un lado, y los obreros y empleados, artesanos y comerciantes, por el otro (p. 108). Esta ruptura es observable a nivel territorial, dado que los cuadros y las profesiones intermedias se han concentrado en las zonas urba- 
nas, mientras que los obreros y artesanos refluyen hacia las zonas rurales. "En consecuencia, la fecundidad es más elevada fuera de las ciudades y las familias son más numerosas en el ámbito rural profundo" (p. 109).

En 2011 el índice de fecundidad galo era, en promedio, de 2.01 hijos por mujer. No obstante, mientras el índice de las mujeres inmigrantes es de 2.75 hijos, el de las mujeres nacidas en Francia es de 1.91 hijos. De la misma forma, la fecundidad es inversamente proporcional a la posición ocupada en la escala social (p. 110). En ese sentido, la mayor diferencia entre inmigrantes y no inmigrantes en esta materia se observa entre los empleados, seguido por los obreros, artesanos y comerciantes, y, finalmente, por los cuadros directivos. En realidad, nos dice el autor, "la inmigración tiende a reforzar ligeramente las diferencias de fecundidad por clase social, en razón de las diferencias de nivel educativo" (p. 111). A ese propósito, existe una gran simetría entre hombres y mujeres, tanto en lo que se refiere a la nacionalidad y al origen como en lo que alude a la categoría social (p. 113).

En lo que afecta a la fecundidad de las mujeres según su categoría social, son las obreras las que tienen la menor tasa, seguido por los cuadros directivos y las profesiones liberales, mientras que las clases medias tienen la más elevada (p. 114). "Para los hombres pertenecientes a las categorías medias y superiores, la fecundidad tiende a aumentar a medida que sus esposas tienen un estatus social más elevado. Sucede todo lo contrario con los obreros y empleados" (p. 117). Le Bras constata que "los hombres adoptan la fecundidad de sus cónyuges, independientemente de su propia categoría social. En otros términos, la mujer decide el número de hijos en función de su situación social y sin realmente tener en cuenta la de su cónyuge" (p. 119).

En ese sentido, se ha producido una profunda mutación durante el último cuarto de siglo, que describen los censos de 1985 y 2011. A lo largo de ese periodo, las mujeres, al adquirir el control técnico de su fecundidad gracias a la píldora y al aborto, se han empoderado y deciden sobre la construcción familiar y, por lo tanto, sobre el número de hijos que quieren tener. El caso más llamativo es el de las obreras ya que, mientras eran las más fecundas en 1985, se han convertido en las menos fecundas en 2011 (pp. 119120). Al contrario, las mujeres inactivas son las que tienen la mayor fecundidad, sabiendo que son a menudo las que carecen de titulación académica.

Asimismo, el nivel educativo de las mujeres incide de manera diferenciada sobre su fecundidad en función de su origen geográfico. Así, mientras que la elevación del nivel educativo de las inmigrantes se traduce en una disminución de su fecundidad, pasando de 2.1 a 1.75 hijos por mujer, la fecundidad de las mujeres nacidas francesas evoluciona en sentido contrario, pasando de 1.3 a 1.7 hijos por mujer (pp. 130-133). En otros términos, 
"mientras que la fecundidad de las mujeres inmigrantes activas sin títulos académicos supera en 50\% la de las mujeres nacidas en Francia, las más calificadas de ambos grupos se encuentran en una situación similar” (p. 133). Esto significa que, si se mide el grado de integración de las mujeres inmigrantes en función de su fecundidad, el nivel educativo resulta ser un factor determinante.

De manera general, Le Bras extrae cinco conclusiones de todo lo anterior (pp. 134-135):

- La fecundidad de las mujeres activas es dos veces inferior a la de las mujeres inactivas, sea cual sea su nivel educativo y origen.

- La fecundidad de las mujeres inmigrantes activas disminuye con el nivel de estudios.

- La fecundidad de las mujeres nacidas francesas aumenta con el nivel educativo.

- La proporción de mujeres inmigrantes disminuye cuando el nivel educativo aumenta.

- La proporción de mujeres activas se incrementa con el nivel educativo, sea cual sea el origen.

Estas cinco constataciones se confirman en la práctica en la totalidad de los departamentos galos, aunque existan variaciones territoriales que son relativamente estables en el tiempo (p. 139). Por ejemplo, Le Bras observa que la fecundidad de las mujeres fluctúa notablemente entre las zonas rurales y urbanas. Por ejemplo, en promedio es de 1.92 hijos por mujer en las zonas rurales y de 1.56 hijos por mujer en las urbanas. Eso se debe al hecho de que el mapa rural se parece mucho al mapa religioso, ya que las tradiciones y prácticas religiosas se mantienen en mayor medida en los pueblos ( $\mathrm{p}$. 144). Las diferencias son igualmente regionales dado que el mencionado mapa “opone una región Sur-Oeste donde, bajo el Antiguo Régimen, el ciclo de vida familiar se organizaba sobre el modelo de la 'familia cepa', en el cual un solo heredero retomaba la explotación familiar. Al contrario del Norte y del Este donde las herencias eran repartidas de manera igualitaria entre los hijos del difunto" (p. 144). En ese sentido, a nivel de los departamentos, y más generalmente de las grandes regiones, las variaciones de fecundidad reflejan comportamientos más antiguos que corresponden a concepciones diferentes de la vida familiar, de la sucesión y de las relaciones intergeneracionales (p. 147).

En el capítulo ulterior, centrado en el desempleo, el demógrafo galo recuerda que tres factores inciden especialmente en éste: la edad, el nivel 
educativo y el origen geográfico. De hecho, los jóvenes y los mayores son las primeras víctimas del desempleo. Más precisamente, observa que "el desempleo es más elevado entre los jóvenes, antes de decrecer hasta aproximadamente los 55 años, y aumentar ligeramente hasta el final de la vida laboral" (p. 156). Asociado a la edad, la ausencia de título académico dificulta sobremanera la entrada y posterior permanencia en el mercado de trabajo, mientras que la posesión de título protege ampliamente del desempleo. Por último, los inmigrantes padecen un mayor nivel de desempleo que los autóctonos, y la diferencia al respecto entre los inmigrantes y los autóctonos tiende a aumentar con la edad y el título académico (p. 164). De hecho, a partir de los 45 años la tasa de desempleo de los inmigrantes duplica la de los no inmigrantes para todos los niveles de estudios (p. 165). Y la variación de las tasas de desempleo de los inmigrantes en función del nivel educativo (entre el 9 y el $23 \%$ ) es menor que la de los autóctonos (entre el 3.7 y el 33.1\%).

A su vez, "la tasa de desempleo de las parejas cuyos miembros son activos varía mucho según la categoría social de los cónyuges” (p. 173). Y la diferencia entre el desempleo de los hombres y el de las mujeres es solamente del $1 \%$ en los hogares de cuadros directivos, pero alcanza el $6.9 \%$ en las familias de obreros. En ese sentido, cuando las mujeres retroceden en la jerarquía social, aumentan las desventajas que padecen en el mercado laboral, mientras que la tasa de desempleo de los varones de una categoría social determinada es menor cuando su cónyuge pertenece a la misma categoría (p. 176). En otros términos, "la distancia social entre los cónyuges aumenta el riesgo de desempleo del hombre, que ocupa una posición inferior o superior, [mientras que] no sucede lo mismo con la mujer [...] [puesto que] sea cual sea su categoría, el riesgo de desempleo es menor si vive con un hombre que trabaja en una profesión intermedia" (pp. 176-177).

Una diferencia social aparece entre las parejas homógamas ya que, en las clases medias y superiores, la homogamia reduce el riesgo de desempleo para ambos miembros de la pareja, mientras que en las categorías populares, las tasas de desempleo son un poco superiores a la media (p. 177). En el primer caso, trabajar en la misma categoría social permite resistir mejor el desempleo porque refuerza la red social y, por lo tanto, las posibilidades de recolocación. Al contrario, en el segundo caso, el hecho de que la pareja esté alejada de las clases medias y superiores incrementa su fragilidad en el mercado laboral, limitando las alternativas en caso de despido (p. 177). En ese sentido, el riesgo de contagio del desempleo en el seno de la pareja es dispar y disminuye con la elevación en la escala social.

Además, en materia de desempleo las diferencias de nivel educativo son paralelas a las diferencias de clase social, sin olvidar la incidencia del 
género, teniendo en cuenta que los hombres se encuentran en promedio en situaciones sociales más elevadas que las mujeres, mientras que estas últimas tienen de media un nivel educativo superior al de sus cónyuges (p. 180). El demógrafo galo observa que "la diferencia relativa entre la tasa de desempleo de las mujeres y la de sus cónyuges se incrementa con el nivel educativo de la pareja, sea cual sea el nivel educativo de la mujer" (p. 180). Esto significa que la mayor vulnerabilidad de las mujeres hacia el desempleo existe independientemente de sus cónyuges ya que, según su nivel de estudios, sus tasas de desempleo superan a las de los hombres del mismo nivel entre el 50 y el 90\% (pp. 181-182). Por lo tanto, "la dominación femenina en la educación no se ha materializado todavía en el mercado laboral. [...] La desigualdad de género ante el desempleo está poderosamente estructurada para resistir a la dominación que las mujeres han adquirido en materia educativa" (p. 182). De la misma forma, tanto para los hombres como para las mujeres, la tasa de desempleo aumenta, en ese orden, conforme se trate de personas nacidas en Francia, de franceses de origen inmigrante y de extranjeros inmigrantes.

Por último, el autor se detiene sobre la onda expansiva del desempleo en el hogar, sabiendo que las personas sufren el que padecen ellos mismos, pero también el desempleo de los familiares que viven en la misma vivienda, ya que comparten un presupuesto común (p. 195); más aún sabiendo que este problema afecta al $10.5 \%$ de los individuos, al $17 \%$ de los hogares y al $62 \%$ de los parientes próximos de estos hogares. Esto se percibe en las indemnizaciones cobradas por las personas, la ayuda social recibida por las familias y la sensación general de inseguridad de los parientes próximos (p. 209). Es obvio que la onda expansiva del desempleo es mayor cuando la pareja se ve afectada directamente, mientras que se atenúa a medida que concierne a una persona alejada.

De manera general, se observa que la progresión de las tasas de desempleo es paralela a la fragilización de las familias y viceversa, de modo que los más protegidos (que disponen de una doble renta) resisten mejor, seguidos de las personas aisladas y las parejas en las cuales solamente un miembro es activo (p. 198). Más detalladamente, la proporción de hogares complejos que tienen al menos un desempleado en su seno se eleva al $29 \%$ y la de los hogares nucleares con una pareja asciende al $18 \%$, justo por debajo de las familias monoparentales $(19 \%)$ y claramente por encima de los hogares aislados (12.5\%). Y si el rol de la edad es menor cuando se analiza el desempleo de los hogares, el de las diferencias sociales sigue siendo notable porque "cuando se desciende en la escala social, la progresión del desempleo es rápida y regular, sea cual sea la forma del hogar” (p. 204). 
Al término de la lectura de Anatomie sociale de la France, conviene subrayar que se trata de uno de los mejores libros de Hervé Le Bras, tanto por las conclusiones extraídas (en materia de pareja, fecundidad y desempleo), los métodos cuantitativos utilizados, como por las modalidades de exposición que asocian claridad, simplicidad y rigor; todo ello ilustrado por innumerables cuadros, gráficas y esquemas. A su vez, el autor hace gala de un gran dominio de los métodos estadísticos que le permiten, sobre la base de los datos ofrecidos por el censo de 2011, contemplar todas las opciones y ofrecer una imagen compleja y matizada de la sociedad gala. Y, al final del libro, añade dos anexos de carácter metodológico relativos por ejemplo a la medida de la variabilidad de los comportamientos. Precisamente, el único reproche que puede hacerse a esta relevante obra, cuya lectura es indispensable para comprender la sociedad gala contemporánea, es la cantidad excesiva de información relacionada con aspectos metodológicos en lugar de centrarse en los resultados del estudio.

\section{Bibliografía}

Le Bras, H. (2014), Atlas des inégalités, París, Autrement.

Le Bras, H. (2015), Le pari du FN, París, Autrement.

Le Bras, H. (2016), Le nouvel ordre électoral. Tripartisme contre démocratie, París, Seuil.

Le Bras, H. y E. Todd (2013), Le mystère français, París, Le Seuil. 
